\title{
Développement de la radiographie $X$ éclair et de l'imagerie de fluorescence induite par $X$ pour la caractérisation de milieux fluides
}

\author{
E. Robert, L. Huré, C. Cachoncinlle, R. Viladrosa et J.-M. Pouvesle
}

GREMI-ESPEO, FR EPEE 0776, UMR 6606 du CNRS, Université d'OHéans, BP. 6744, 45067 Orléans cedex 2, France

\begin{abstract}
Résumé : Nous présentons les motivations du développement de deux nouveaux diagnostics à base de photons $\mathrm{X}$ à $10 \mathrm{keV}$, radiographie éclair et imagerie de fluorescence induite par $\mathrm{X}$. Le principe de ces deux techniques, les dispositifs expérimentaux utilisés incluant la source flash $\mathrm{X}$ dédiée à ces études sont décrits. Les premières expériences de faisabilité et de validation de ces diagnostics pour la caractérisation d'écoulements gazeux à haute pression sont présentées. Les développements des deux techniques et nos récents travaux sur des sprays sont finalement discutés.
\end{abstract}

\section{CONTEXTE}

Il existe aujourd'hui un besoin clairement identifié de données expérimentales permettant de caractériser l'écoulement de fluides dans des conditions physiquement contraignantes. Cette recherche de diagnostics s'exprime par exemple dans les études liées à l'injection de carburant à haute pression, l'atomisation de sprays liquides et la combustion turbulente à très haute pression dans les moteurs cryogéniques des lanceurs de fusées. Ces applications se caractérisent par l'existence de zones d'écoulement à haute pression (jusqu'à plusieurs centaines de bars), à haute température (jusqu'à plusieurs milliers de degré). De plus, ces milieux sont souvent la source d'émissions lumineuses intenses (flamme de combustion). De nombreuses techniques de mesures optiques (Particle Image Velocimetry, Laser Doppler Anemometry, Phase Doppler Anemometry, Laser Induced Fluorescence) ont été développées. Ces diagnostics fournissent des résultats remarquables pour la mesure des profils de densités, de champ de vitesse, de morphologie spatio-temporelle des écoulements. Cependant dans le cas des milieux les plus complexes, leur mise en cuvre devient particulièrement délicate et les zones les plus denses (sortie des injecteurs) ou les plus lumineuses (phase initiale de combustion) ne peuvent plus être caractérisées du fait de limitations physiques intrinsèques telles que dispersion, diffusion, émission parasite, etc...

Une des voies étudiée pour pallier à ces difficultés consiste à exploiter le fort pouvoir de pénétration des rayons $X$. Nous développons au GREMI deux méthodes, radiographie éclair et imagerie de fluorescence induite (IdeFIX), basées sur l'absorption d'une partie du flux de rayonnement X émis par une source compacte et impulsionnelle appelée flash X développée et optimisée au laboratoire.

Dans la suite de ce papier, nous présentons le principe des deux diagnostics et leur première application à la caractérisation de l'écoulement de jets d'argon dans l'air ambiant. La comparaison et les spécificité propres à chacune des deux techniques sont résumées. L'exploitation plus approfondie des clichés radiographiques ainsi que nos récentes expériences d'imagerie $2 \mathrm{D}$ de fluorescence résolues à la fois spectralement et temporellement sont ensuite discutées. Finalement, nous évoquerons les travaux menés en collaboration avec le Laboratoire de Combustion et Systèmes Réactifs (LCSR) à Orléans dans le cadre de la fédération de recherche EPEE sur la caractérisation de sprays liquides.

\section{PRINCIPE DES DIAGNOSTICS-DISPOSITIFS EXPERIMENTAUX}

La radiographie consiste à illuminer un objet à l'aide de rayons $\mathrm{X}$ et à recueillir une projection à deux dimensions du profil d'absorption du volume traversé. Les écarts d'absorption du milieu et de son environnement ou des zones de densité et/ou d'épaisseur variables du milieu se traduisent par l'enregistrement de niveaux de gris différents. Bien que largement exploitée dans différents domaines, la radiographie des milieux à haute pression constitués d'éléments de numéro atomique faible $(\mathrm{H}, \mathrm{N}, \mathrm{C}, \mathrm{O})$ requiert l'utilisation de photons $\mathrm{X}$ d'énergie située dans la gamme $5-10 \mathrm{keV}$ pour lesquels l'absorption devient significative (qqes. \%). Nous avons développé au GREMI une source adaptée à ce besoin qui 
émet de fortes doses (jusqu'à $1 \mathrm{R}$ à la fenêtre de sortie) essentiellement dans les raies caractéristiques du fer et du tungstène (gamme 6-12 keV). Notre source produit de plus des impulsions rapides (typiquement $20 \mathrm{~ns}$ ) ce qui permet d'effectuer des études de cinéradiographie ou de photoexcitation rapides.

Lors de l'irradiation d'un milieu, particulièrement gazeux, par des photons $\mathrm{X}$, il peut se produire une émission de fluorescence, souvent dans le domaine UV, désigné ci après Fluorescence Induite par X ou FIX. Nous avons mené une étude systématique de FIX de cibles gazeuses à des pressions multiatmosphériques de gaz rares purs, en mélange binaire ou dilués avec de l'azote, oxygène, air...

L'IdeFIX consiste dans la mise en place d'une expérience d'imagerie de cette FIX. Dans les travaux d'IdeFIX présentés ici, nous avons employé des lentilles, des filtres en longueurs d'onde et des détecteurs intensifiés synchronisés avec les impulsions de rayonnement $\mathrm{X}$.

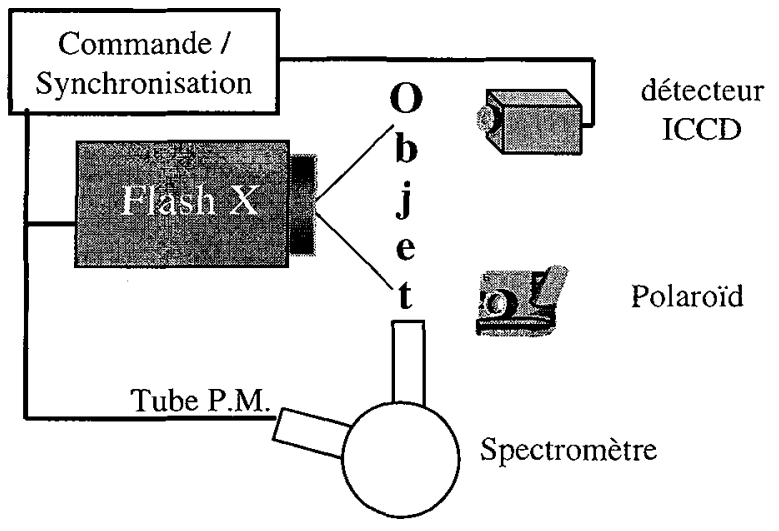

Figure 1 :Dispositif expérimental pour la radiographie, la FIX et l'IdeFIX

La figure 1 schématise les différents dispositifs expérimentaux utilisés. Nous retrouvons la source d'excitation, flash X, "l'objet" à diagnostiquer, les films pour la radiographie et un détecteur couplé à un dispositif d'imagerie pour l'imagerie de FIX. Nous effectuons par ailleurs une analyse sytématique par spectroscopie d'émission résolue en temps des éventuelles FIX dans les objets que nous étudions.

\section{DIAGNOTICS DES JETS GAZEUX}

Nos premières expériences de faisabilité de radiographie et d'IdeFIX ont été réalisées sur des jets d'argon s'écoulant dans l'air à la pression atmosphérique au travers de buses de dimensions millimétriques. Ces jets ont été choisis comme éprouvette pour deux raisons essentielles. D'une part des épaisseurs millimétriques d'argon à la pression atmosphérique correspondent à une absorption de quelques \% à $10 \mathrm{keV}$, typique dans les applications envisagées. Par ailleurs, nous avons préalablement étudié le spectre de FIX de ces écoulements [1], celui ci est constitué de fluorescence de l'argon mais aussi d'espèces de l'environnement du jet $\left(\mathrm{N}_{2}{ }^{*}\right.$ et $\left.\mathrm{OH}^{*}\right)$ peuplées par transfert collisionnel. Sur la figure 2 les profils de niveaux de gris extraits d'une radiographie de deux jets d'argon croisés ainsi que les profils de fluorescence enregistrés sur ce même écoulement sont présentés pour différentes distances par rapport au plan de sortie des buses. On note dans les deux cas, la présence de deux pics témoins de la présence de l'argon en sortie de buses. Ces deux pics se rapprochent pour n'en former plus qu'un plus large au point de croisement des deux jets vers $10 \mathrm{~mm}$. Ces résultats attendus permettent de confirmer l'intérêt de l'utilisation d'une source émettant autour de $10 \mathrm{keV}$ pour la caractérisation de milieu à faible absorption par radiographie. Il s'agit par ailleurs à notre connaissance des premiers résultats de diagnostic par IdeFIX. Cette expérience permet de valider la technique d'IdeFIX comme outil de diagnostic du fait de la bonne concordance des données obtenus par l'emploi de cette technique en comparaison avec le radiographie. D'une manière générale, les deux techniques peuvent être employées simultanément ou indépendamment selon leur sensibilité respective. A titre d'exemple, l'étude de l'écoulement de jets d'argon d'un millimètre d'épaisseur n'a pas été possible dans nos conditions expérimentales par 
radiographie (contraste sur films trop faible) tandis que des résultats de même qualité que ceux présentés sur la figure 2 ont été enregistrés par IdeFIX dans ce cas. Il existe bien sûr à l'inverse, des situations où l'objet est parfaitement opaque aux $\mathrm{X}$ de $10 \mathrm{keV}$ mais ne présente aucune FIX.

\section{Radiographie}

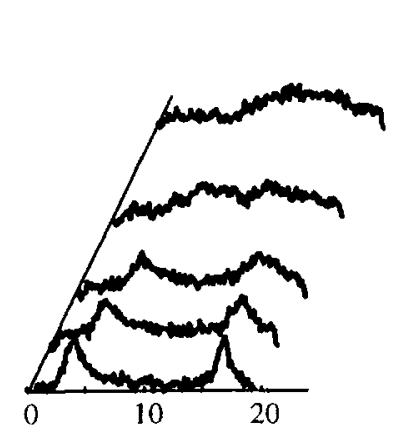

Position sur le détecteur (mm)

\section{Fluorescence Induite par X}

Distance aux buses (mm)
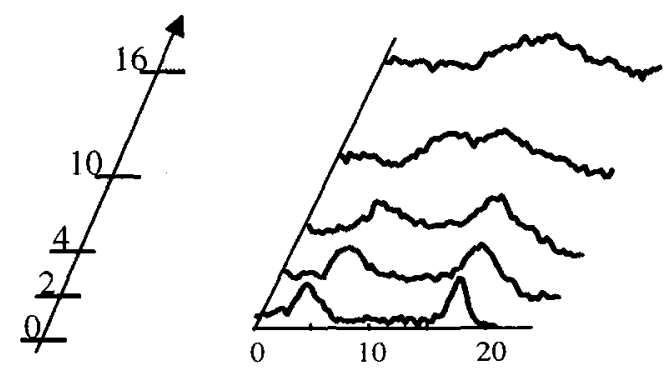

Position sur le détecteur $(\mathrm{mm})$

Figure 2 :Diagnostic par radiographie et imagerie 1D de FIX de jets d'argon dans l'air

\section{DEVELOPPEMENTS}

L'obtention de données quantitatives (densité, épaisseur, dimension de motifs...) à partir des clichés radiographiques nécessite l'étude préalable de l'influence de certains paramètres inhérents à la technique. Nous avons ainsi vérifié la linéarité des films en fonction de la dose reçue, apprécié l'importance de la taille du foyer émetteur, de l'énergie des $X$ incidents sur le contraste et la résolution spatiale. La connaissance de ces caractéristiques et l'inversion par un algorithme développé dans l'équipe de la projection 2D du profil d'absorption en volume nous ont permis d'obtenir des mesures quantitatives de densités dans des écoulements avec des résolutions de l'ordre de la centaine de $\mu \mathrm{m}$ [2]. Par ailleurs, l'emploi de demi-lentilles $\mathrm{X}$ à base de polycapillaires, dont l'un des foyers est placé en coïncidence avec le foyer du flash $X$, nous a permis de mesurer des contrastes nettement supérieurs lors d'expériences en éclairement $X$ parallèle en comparaison à ceux obtenus avec notre source divergente. Ces premières expériences permettent d'envisager des diagnostics par plans de rayons $\mathrm{X}$, proches des techniques de tomographie optique et facilitant de plus la reconstruction des profils tridimensionnels.

Nous avons récemment effectué des expériences d'IdeFIX filtrée en longueur d'onde sur des jets d'argon à la pression atmosphérique. Ainsi la figure 3 présente les images et les profils de fluorescence obtenus dans différentes gammes spectrales sur un jet d'argon cylindrique (diamètre $1 \mathrm{~cm}$ ) s'écoulant dans l'air ambiant. Sur la figure $3 \mathrm{a}$ ) on observe un profil symétrique centré sur l'axe du jet et d'une largeur d'environ $1 \mathrm{~cm}$, sur la figure $3 \mathrm{~b}$ ) enregistrée en intercalant entre le jet et le détecteur un filtre permettant de n'observer que la fluorescence propre à l'argon on mesure également un profil symétrique centré sur l'axe. En revanche sur la figure $3 \mathrm{c}$ ), enregistrement de la fluorescence de l'azote, on observe un profil symétrique mais présentant deux pics situés en périphérie du jet. Ce résultat témoigne du transfert d'excitation de l'argon vers l'azote en périphérie du jet. Il faut souligner ici que l'irradiation par flash $\mathrm{X}$ de l'air ambiant à la pression atmosphérique ne conduit à la production d'aucune FIX. Cette première expérience d'IdeFIX filtrée en longueur d'onde permet de démontrer la faisabilité de caractérisation de mélange ou de zones d'interfaces entre deux écoulements.

Des expériences d'IdeFIX résolues temporellement sont également d'ores et déjà envisageables : le filtrage spectral pourrait ainsi être réalisé par la sélection de fenêtres temporelles adaptées à chacune des espèces à diagnostiquer. Dans le cas des jets d'argon dans l'air on peut ainsi souligner que la fluorescence de l'argon reproduit temporellement l'impulsion X (20 ns) tandis que les transitions dans l'azote ou pour le radical $\mathrm{OH}^{*}$ durent quelques centaines de nanosecondes. 

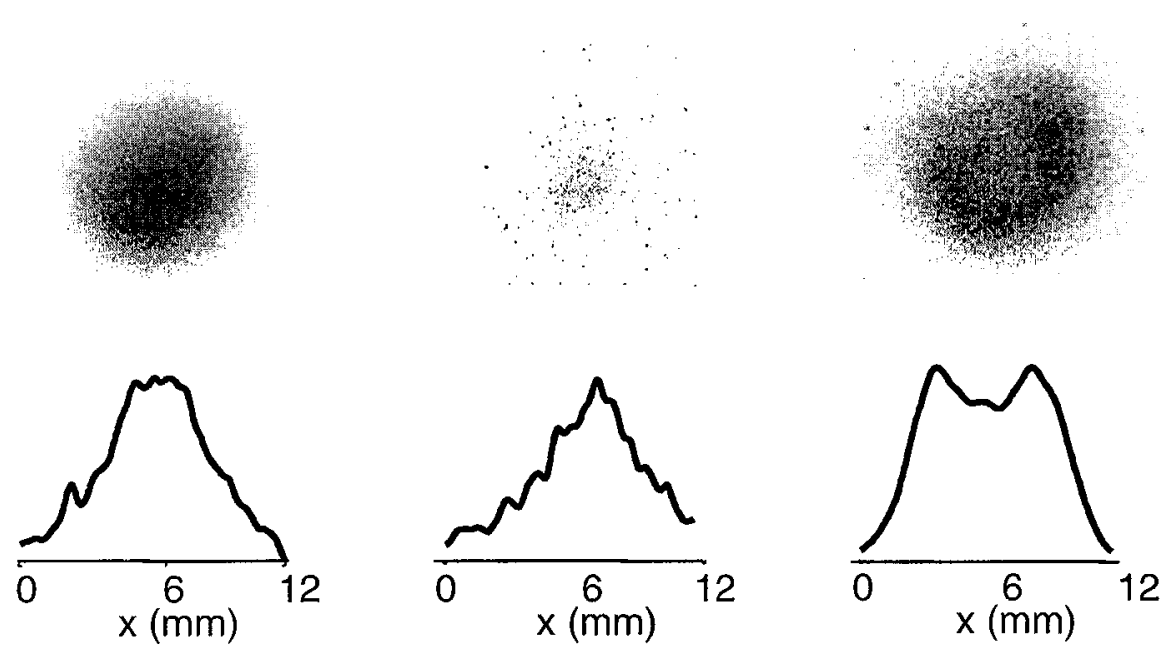

Figure 3 :Imagerie de FIX filtrée en longueur d'onde : a) fluorescence globale (200-800 nm), b) fluorescence de l'argon (200$250 \mathrm{~nm})$ et c) fluorescence de I'azote $(280-380 \mathrm{~nm})$

La figure 4 illustre les travaux entrepris dans le cadre de la fédération EPEE en collaboration avec le LCSR. Nous cherchons à adapter nos diagnostics $X$ à la caractérisation de sprays. Ces études sont liées aux travaux consacrées à la combustion de carburant dans les moteurs à haute pression en mode turbulent. Nos expériences de radiographie ont permis d'effectuer des études paramétriques de l'évolution de la taille de la zone liquide en sortie d'injecteur (dite dard), de l'angle d'ouverture du spray, du diamètre et de la densité de gouttes liquides... en fonction des débits d'air et d'eau, du volume de la chambre d'atomisation. La réalisation d'une cellule d'injection de gaz jusqu'à 5 bars dans une ambiance contrôlée couplée à des mesures par spectroscopie, radiographie et IdeFIX est en cours de réalisation.

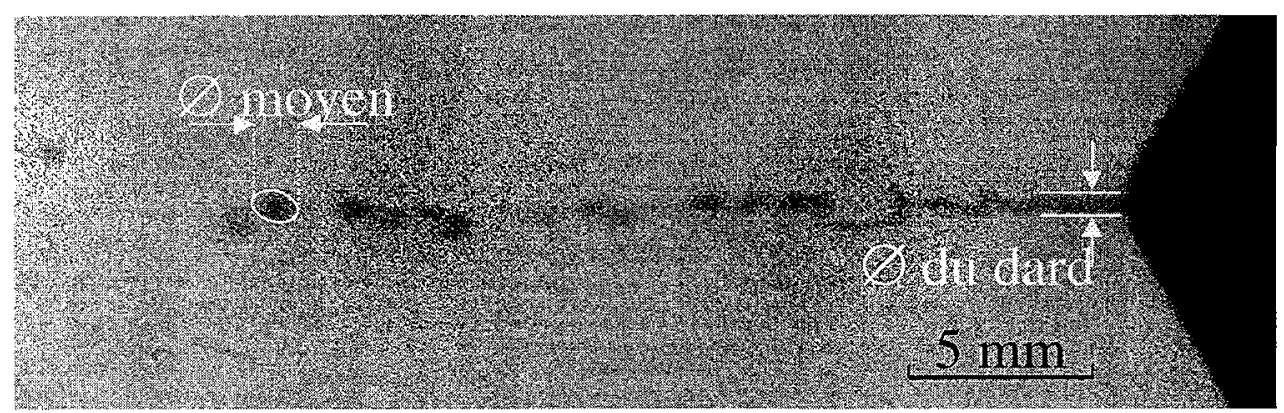

Figure 4 :Radiographie sur un tir d'un spray d'eau atomisé par air.

\section{CONCLUSION}

La faisabilité et le développement de diagnostic par radiographie et imagerie de fluorescence induite par $\mathrm{X}$ à $10 \mathrm{keV}$ ont été réalisés sur des jets gazeux à la pression atmosphérique et pour des écoulements diphasiques. L'obtention de données quantitatives par radiographie monocoup (20 ns) ainsi que l'application de l'IdeFIX filtrée spectralement ouvrent des perspectives prometteuses à ces nouveaux diagnostics.

\section{Références}

1. L. Huré, E. Robert, C. Cachoncinlle, R. Viladrosa, J.M. Pouvesle, J.Phys. IV, 9, Pr5-83 (1999).

2. L. Huré et al., ce volume. 